\title{
Première femme médecin suisse - Dr. med. Marie Heim-Vögtlin
}

\section{Judith Naef}

lic. iur., avocate, directrice mws medical women switzerland-femmes médecins suisse

Le 7 octobre 1845 naît à Bözen AG une petite fille pétillante, avide de savoir et pleine de tempérament: Marie Vögtlin. C'est la deuxième fille du pasteur du village. Elle a le privilège de pouvoir jouir d'une formation, mais souffre des conventions auxquelles elle doit se soumettre en tant que femme du XIX ${ }^{\mathrm{e}}$ siècle. En dépit de nombreuses résistances, elle les chamboulera finalement (presque) toutes.

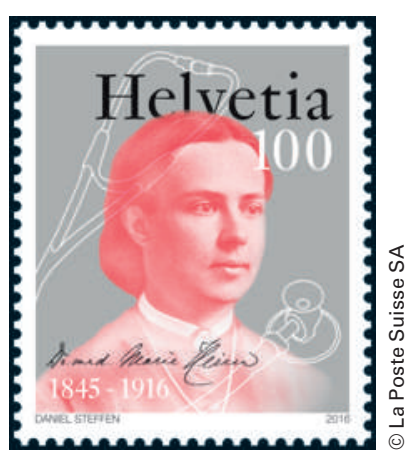

Sur l'initiative de la mws medical women switzerland femmes médecins suisse, la Poste Suisse édite un timbreposte spécial en I'honneur du $100^{\mathrm{e}}$ anniversaire du décès de la première femme médecin suisse.

\section{Force de caractère et père prévoyant}

Soutenue par son père, Marie Vögtlin est la première Suissesse à être acceptée en faculté de médecine à l'Université de Zurich en 1868, ce qui provoque - sans trop de surprise - un scandale au niveau national. Ce n'est pourtant qu'une demie victoire, car des études sans maturité ne lui servent à rien. Sans maturité, elle n'a pas le droit de travailler comme médecin (sic!). Encore une fois, son père doit intervenir afin que pendant ses études de préparation à l'«examen d'Etat médical propédeutique» elle puisse, avec une autorisation spéciale du conseil d'Etat, être la première fille à obtenir sa maturité en Argovie. Elle passe son examen d'Etat avec brio en 1870. Mais une jeune fille comme Marie Vögtlin ne va pas se contenter de ce succès: elle sera la première femme en Europe à se spécialiser en gynécologie et en obstétrique à Leipzig et à Dresden. En 1874, elle sera finalement la première Suissesse à obtenir son doctorat en médecine à l'Université de Zurich. L'ouverture de son cabinet la même année à Zurich nécessitera toutefois de nouveau l'intervention de son père.

\section{Epouse et mère - un exemple pour ses contemporaines}

En 1875, une année après l'ouverture de son cabinet, Marie épouse le professeur de géologie Albert Heim. Le couple renonce à avoir des enfants jusqu'à ce que la carrière de Marie soit assurée. Elle accouche finalement de leur premier enfant à l'âge de 36 ans, ce qui pour son époque est très tard. Deux enfants suivront. Malgré sa profession de femme médecin, Marie Heim-Vögtlin ne peut pas empêcher que leur troisième enfant meurt à l'âge de trois semaines seulement.

Marie Heim-Vögtlin restera active professionnellement durant toute sa vie. Vu que selon la loi de l'époque, la femme était sous la tutelle de son mari, il lui faudra toutefois l'autorisation de son mari. Le mari pouvait même disposer librement d'un éventuel salaire de son épouse. Mais Albert Heim se montre généreux et compréhensif et permet à son épouse, parfois avec quelques soupirs, d'exercer sa profession et de garder son revenu. Il ne s'imaginait certainement pas, en tant que patriarche bien intentionné, que cette décision aiderait un jour à sauver l'honneur de sa famille d'origine et par conséquent son propre bon nom. Le fait est toute-

\section{-2558- Dr. Med. Marie vögtlin, Arzt für Geburtshülfe und Frauenkrankheiten, Hottingerstrasse Nr. 424. \\ Sprechstunde täglich von $10-12$ Uhr.}

Annonce dans le Tagblatt de la Ville de Zurich du 12 juillet 1874 [1]. 


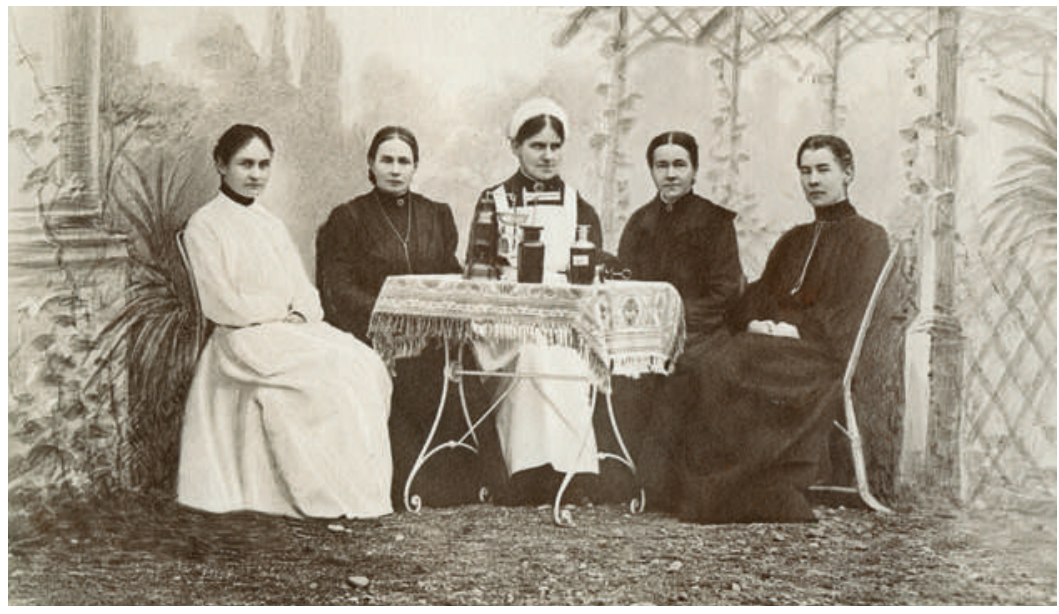

Femmes remplissant des fonctions de direction. La direction de l'école d'infirmières suisse vers 1910 (de gauche à droite): Dr. med. Anna Baltischwiler, Dr. med. Marie HeimVögtlin, Supérieure Ida Schneider, Dr. med. Anna Heer, Dr. med. Jenny Thomann-Keller (C) Fondation Gosteli, archives, Worblaufen)

fois que les dettes du père d'Albert Heim, résultant de la faillite d'une propre caisse d'épargne, ne pourront être remboursées que grâce au revenu supplémentaire de Marie.

«Pour de nombreux contemporains, Marie et Albert Heim sont un exemple à suivre et porteurs d'espoir représentant l'idéal du couple moderne. C'est la raison pour laquelle ils vivent dans une sorte de vitrine, et sous une énorme pression de la réussite. Leur relation devait tenir bon, car aux yeux de Marie, un échec aurait discrédité toutes les études pour les femmes, et les conservateurs rétrogrades se seraient fait un malin plaisir à manifester ouvertement leur satisfaction" [2]. Autant Albert Heim se montrait tolérant face à l'activité professionnelle de son épouse, autant il s'opposait violemment au droit de vote des femmes, car il ne voulait pas déranger "le travail de qualité des femmes ... ni le freiner par l'introduction de cette nouvelle obligation» [3].

\section{Sens politique}

Marie Heim-Vögtlin - comme d'autres pionnières de sa génération - est passée maître en matière de comportement progressif dans un environnement conservateur. Dans les moments critiques, elle fait appel à des hommes influents pour aider à faire passer des projets de femmes, malgré un vent social ou politique contraire. C'est ainsi qu'un conseiller municipal est à la tête de la commission de construction pour l'école d'infirmières, dont fait aussi partie le mari de Marie, Albert Heim. Malgré une obstination et un besoin d'indépendance hors du commun, Marie Heim-Vögtlin sait, aussi dans son environnement professionnel, se soumettre aux conventions quand la cause lui semble sensée. Elle ne soigne par exemple pas les hommes, et lors d'opérations délicates, elle demande à un confrère de l'assister pour qu'il soit témoin d'un traitement irréprochable! De cette manière, elle protège sa réputation et permet à d'innombrables femmes un traitement professionnel. Contrairement à la deuxième femme médecin suisse, Caroline Farner, sa réputation et son image resteront irréprochables toute sa vie par cette pratique habile, malgré la rupture de nombreuses conventions.

\section{Précurseuse et pionnière}

Marie Heim-Vögtlin se distingue par une force de caractère hors du commun. Mère de famille active professionnellement, elle lutte sans répit pour améliorer la situation de la population et se bat pour la formation des femmes et le droit de vote des femmes. Elle inspire de nombreuses contemporaines qui à leur tour sortent de l'ombre de leur époux.

Marie Heim-Vögtlin est consciente de l'importance et de la force d'un réseau de personnes qui défendent les mêmes idées, et soutient par conséquent divers groupements de femmes. Elle a particulièrement à cœur la ligue de tempérance. Elle est co-initiatrice de la Ligue suisse des femmes abstinentes (SBAF). Elle soutient aussi depuis le début le projet de la Société d'utilité publique des femmes suisses (SFG) visant à fonder à $\mathrm{Zu}$ rich un hôpital pour les femmes, avec une école d'infirmières intégrée. Appelée la «Pflegi», cet établissement deviendra le premier hôpital en Suisse dirigé par des femmes et offrira des postes de travail et de formation à des femmes médecins. Uniquement des femmes y sont soignées. Et les élèves infirmières reçoivent pour l'époque une excellente formation dans cet établissement de confession neutre. Le rôle important que Marie Heim-Vögtlin joue dans la fondation de l'hôpital se constate au fait que la première pierre sera posée en son honneur le jour du 25 anniversaire de son examen de doctorat le 11 juillet 1899. Dès le début, Marie HeimVögtlin en est la trésorière. Par contre, la directrice est la Dr. med. Anna Heer, qui a terminé ses études de médecine en 1892 à Zurich. L'influence de Marie HeimVögtlin a laissé des traces visibles aujourd'hui encore et mérite dans une large mesure d'être qualifiée d'avantgardiste.

\section{Visions et faits au-delà de la mort}

Dr. med. Marie Heim-Vögtlin décède le 7 novembre 1916 à l'âge de 71 ans d'une tuberculose pulmonaire. Elle ne pourra malheureusement plus voir se réaliser son désir d'autoriser les femmes médecins à participer aux programmes de formation post-graduée des médecins. 
En ce temps-là, des programmes de formation postgraduée sont déjà régulièrement proposés aux médecins praticiens, et sont déjà autant important qu'aujourd'hui pour leur développement professionnel et les contacts entre eux. Ce bastion restera encore longtemps réservé aux confrères. Mais les femmes médecins se montrent ingénieuses: elles s'affilient comme membres individuels à la «Medical Women's International Association» (MWIA). En 1922, elles organisent le premier congrès MWIA à Genève, ce qui donne le coup d'envoi à la fondation de l'association suisse des femmes médecins qui existe aujourd'hui encore sous le nom de mws medical women switzerland - femmes médecins suisse (voir encadré).

\section{Enfants et carrière - le défi reste inchangé}

Marie Heim-Vögtlin est le prototype de la femme médecin et de la mère active professionnellement. Elle lutte à son époque déjà avec des problèmes similaires à ceux des femmes médecins d'aujourd'hui: elle est sollicitée de tous les côtés dans sa vie privée et professionnelle. C'est grâce au soutien et à la compréhension de son entourage, apportés parfois avec quelques grincements de dents, qu'elle pourra développer ses qualités hors de son foyer. lic. iur. Judith Naef

Rechtsanwältin

Directrice mws

medical women switzerland

Stampfenbachstrasse 52

CH-8006 Zurich

naef[at]judithnaef.ch
Conférence annuelle mws avec Assemblée générale:

100 ans de femmes médecins en Suisse de Marie Heim-Vögtlin à nos jours

Samedi 12 mars 2016, de 09h30 à 16h00, Kultur \& Kongresshaus Aarau

La mws rend hommage à la première femme médecin lors de sa prochaine Conférence annuelle et se penche sur les progrès réalisés depuis lors. Un exposé particulier sera consacré au timbre-poste spécial.

La Conférence sera ouverte au public le matin, avec une traduction simultanée en français!

Détails et inscription sur www.medicalwomen.ch

Comme le font de nombreuses femmes de nos jours, Marie relègue son désir d'avoir des enfants au second plan jusqu'à ce que sa carrière et sa réputation de femme médecin soient assurées. Contrairement à la plupart des femmes médecins d'aujourd'hui, elle peut cependant faire appel à du personnel domestique, de telle sorte à ce que les questions de la crèche et du travail à temps partiel pour s'occuper de la famille ne se posent pas.

\section{Précieux réseaux de femmes}

La stratégie de Marie Heim-Vögtlin et de ses compagnes d'armes consistait à se rallier en multiples réseaux. Ces réseaux servaient à des échanges d'idées entre semblables et entre femmes avec des expériences similaires. Mais aussi à s'aider elles-mêmes et à pouvoir s'attaquer à de grands projets et à les réaliser ensemble avec détermination. Les femmes mettaient en place des champs d'action réservés aux femmes au sein desquels celles-ci pouvaient développer leurs talents et leurs aptitudes. Les conditions-cadres pour les femmes médecins ont énormément changé ces 100 dernières années. Faut-il en déduire que l'échange personnel en réseaux serait devenu superflu? Les champs dans lesquels les femmes qui font des expériences semblables se rencontrent et échangent leurs points de vue seraient-ils dépassés et plus d'actualité? Est-ce qu'en 2016, il n'est plus nécessaire que les femmes médecins, pour réaliser des visions communes, réunissent leurs forces et se soutiennent mutuellement? La culture de management dans les établissements de santé a-t-elle enregistré et intégré les impulsions féminines émises depuis plus de 100 ans? Nous pensons que non: même s'il a eu un progrès énorme, il reste encore quelques obstacles hauts à surmonter jusqu'à ce que les médecins, hommes et femmes, puissent évoluer dans des conditions de travail qui répondent aux exigences des deux sexes. C'est dans ce sens que mws femmes médecins suisse continue d'agir, aussi en mémoire de Marie Heim-Vögtlin et de ses énormes performances. 